\section{SAT0229 NUMBER NEEDED TO TREAT (NNT): IMPLICATION IN RHEUMATOLOGY CLINICAL PRACTICE}

${ }^{1} \mathrm{M}$ Osiri, ${ }^{2} \mathrm{ME}$ Suarez-Almazor, ${ }^{3} \mathrm{GA}$ Wells, ${ }^{3} \mathrm{P}$ Tugwell. 'Department of Medicine, Chulalongkorn University Hospital, Bangkok, Thailand; ${ }^{2}$ Health Services Research, Baylor College of Medicine, Houston, USA; ${ }^{3}$ Department of Medicine, University of Ottawa, Ottawa, Canada

\subsection{6/annrheumdis-2001.865}

Background A major challenge for rheumatologists is to translate the research data into treatment decision for their patients in clinical practice. One approach that is becoming increasingly used is the ?number needed to treat? (NNT), the reciprocal of absolute risk reduction, which is the number of patients who must be treated in order to obtain the benefit of interest in one new patient.

Objectives To calculate the NNT from rheumatology systematic reviews and clinical trials.

Methods We calculated the NNT from clinically important outcomes in rheumatology from the Cochrane Database of Systematic Reviews in the Cochrane Library Issue 2, 2000 or from the absolute risk differences of the primary outcomes from the original RCTs. If these outcomes were not available, the NNT was calculated from mean improvement of tender joint counts. Key words included musculoskeletal, rheumatology, and systematic reviews.

Results 15 systematic reviews and 11 original articles contributed the data for NNT calculation. The smallest NNT for the patients meeting the ACR20 criteria was from etanercept treatment compared to placebo (NNT 1.8;95\% CI 1.1,4.7). The NNT and 95\% CI for the patients treated with MTX, SSZ and leflunomide was $5.0(3.3,11), 3.6(2.5,6.7)$, and $3.9(2.7,6.6)$, respectively. The NNT for combination treatment (cyclosporin + MTX, infliximab + MTX, and prednisolone + MTX + SSZ) ranged from 2.6 to 4.8 . The NNT for azathioprine $(2.4 ; 95 \%$ CI $1.8,8.9)$ and high dose D-penicillamine $(5.2 ; 95 \%$ CI $2.8,34.2$ ) was calculated from mean change of the number of tender joints. The NNT for meeting the Paulus criteria in antimalarial-treated patients was $4.6(95 \%$ CI 2.5,25.6) and in injectable gold group was 4.0 (2.3,14.3). Among different NSAIDs used in hip OA, only etodolac (NNT 4.4; 95\% CI 2.4,24.4) and tenoxicam (NNT 3.8, 95\% CI 2.5,7.3) showed a significant NNT and 95\% CI for relieving pain over placebo.

Conclusion The NNT is a term translated from the less understandable research data to help clinicians in routine practice decision-making. Comparisons of the NNTs can be done among different treatment options for the same disease and outcome. The NNT should be adjusted for the baseline risk and treatment duration in individual patients.

\section{SAT0230 ECONOMIC ANALYSIS OF ROFECOXIB VERSUS NSAIDS: COMPARISON ACROSS DIFFERENT PROVINCES IN CANADA}

${ }^{1} \mathrm{MA}$ Marentette, ${ }^{2} \mathrm{C}$ Attard, ${ }^{3} \mathrm{JM}$ Pellissier, ${ }^{4} \mathrm{SX}$ Kong, ${ }^{1} \mathrm{C}$ Sauvageau. ${ }^{1}$ Health Economics, Patient Health, Merck Frosst Canada \& Co., Kirkland, QC, Canada; Innovus Research, Inc., Burlington, ON, Canada; ${ }^{3}$ Merck Research Laboratories, Blue Bell, PA, USA; ${ }^{4}$ Merck \& Co., Whitehouse Station, NJ, USA

\subsection{6/annrheumdis-2001.866}

\section{Background}

Objectives Rofecoxib (VIOXX) patients were shown in Phase III clinical trials to have significantly fewer clinically significant gastrointestinal (GI) adverse events than those who received non-selective NSAIDs for the treatment of osteoarthritis (OA). We evaluated the clinical and economic consequences of rofecoxib prescription in each of the ten Canadian provinces for patients over 65 who were assumed to have failed acetaminophen therapy.

Methods A cost-effectiveness analysis was conducted comparing rofecoxib to non-selective NSAIDs with respect to their gastrointestinal effects on patients with OA. A decision analytic model, with a time horizon of one year, was constructed focussing on the event rates from a pooled analysis of $8 \mathrm{Phase} \mathrm{IIb} / \mathrm{III}$ clinical trials. The number of perforations, ulcers and bleeds (PUBs) in each strategy was used as the primary measure of effectiveness. Province-specific inputs were incorporated to determine the relative impact of provincial differences in reimbursement policies, medication mix, prescription length, and dispensing fees on the economic results.

Results In six of the ten Canadian provinces, the replacement of traditional NSAIDs with rofecoxib was associated with fewer PUBs, but at a slightly higher annual cost per patient to the provincial governments (range: \$21.37 - \$133.42). In Alberta, Manitoba, Quebec and Newfoundland the use of rofecoxib was associated with an annual cost saving in addition to the clinical benefit of fewer PUBs. The variation in the model results across provinces was primarily due to the variation in average NSAID and GPA costs across the provinces. As drug choices differ between the provinces, the average daily cost of an NSAID was seen to vary between $\$ 0.49$ in Nova Scotia to $\$ 1.24$ in Newfoundland. Likewise, the average daily cost of a GPA ranged from \$0.72 in New Brunswick to \$2.11 in Alberta.

Conclusion Rofecoxib may represent a cost-effective alternative to non-selective NSAIDs for the treatment of OA in Canada, despite provincial differences in reimbursement policies and medication mix which generate varying burdens on payers and patients.

\section{SAT0231 HINDRANCES IN EFFECTING INSURANCES AMONG DUTCH ANKYLOSING SPONDYLITIS (AS) PATIENTS}

A Van Tubergen, A Boonen, S Van der Linden. Rheumatology, University Hospital Maastricht, Maastricht, The Netherlands

\subsection{6/annrheumdis-2001.867}

\section{Background}

Objectives To evaluate whether AS patients encounter hindrances in effecting different types of insurances after the diagnosis of AS.

Methods In January 2000, a questionnaire was sent to 129 Dutch AS patients inquiring about three different types of insurances: health insurance, additional work-disability insurance (for non-covered loss of income due to illness) and life insurance. We identified patients who had effected one or more insurances after the diagnosis of AS, and had encountered hindrances. Hindrances were defined as obligation to supply medical information, obligation to undergo medical examination, or restrictions in the insurance contract, such as higher premium, no restitution for AS-related problems etc. As a control group served those patients who also effected the above insurances, but before the diagnosis of AS.

Results After diagnosis of AS 15 out of 129 patients had effected a health insurance. Four of them were obliged to supply medical information versus 0 from the control group $(n=114)(p<$ 
0.001); 1 patient had to undergo a medical examination versus 3 from the controls $(p=0.96)$; and for 2 patients restrictions in the contract were imposed versus 0 from the controls $(\mathrm{p}=$ $0.005)$.

19 out of 38 patients effected an additional work-disability insurance after the diagnosis of AS. Six of them were obliged to supply medical information versus 0 from the control group (n $=19)(\mathrm{p}=0.027) ; 2$ patients had to undergo a medical examination versus 0 from the controls $(p=0.47$ ); for 2 patients restrictions in the contract were imposed versus 0 from the controls $(\mathrm{p}=0.47)$.

25 out of 57 patients effected a life insurance after the diagnosis of AS. Seventeen patients were obliged to supply medical information versus 2 from the control group $(\mathrm{n}=32)$ ( $\mathrm{p}<$ 0.001); 6 patients had to undergo a medical examination versus 3 from the controls $(p=0.26)$; for 7 patients restrictions in the contract were imposed versus 1 from the controls $(p=0.02)$.

Conclusion In The Netherlands, AS patients encounter substantial hindrances in effecting insurances. Especially in effecting a health or life insurance medical information is often requested, which may lead to restrictions in the insurance contract.

\section{SAT0232 MANAGEMENT OF CHRONIC MUSCULOSKELETAL PAIN}

M Pileckyte, N Misiuniene. Department Rheumatology, Kaunas Medical University, Kaunas, Lithuania

\subsection{6/annrheumdis-2001.868}

\section{Background}

Objectives Of the study were to explore the management of sufferers with chronic musculoskeletal pain (CMP) syndromes.

Methods A random sample of subjects was selected in the vicinity of two main outpatient clinics of the city Kaunas. All subjects aged 25-74 were requested to indicate on a mannequin any pain which had been lasting at least $24 \mathrm{~h}$ since the last month. Subjects who met the ACR 1990 definition for chronic widespread pain (CWP) and subjects who satisfied the standard definition of chronic low back pain (CBP) but not the ACR definition for CWP were interviewed according to a standard questionnaire. A follow-up screening of all the subjects was carried out after 6 months. Subjects who still reported CWP and CBP were classified as "persistent" CWP and CBP sufferers. Outpatient medical records of all these subjects were analysed.

Results The study population comprised 1,404 subjects, 1,030 being aged $25-74$. The response rate was $83.01 \%$. The average age of respondents was $49.29 \pm 13.09,61.64 \%$ being females. The prevalence of CWP was $18.60 \%$ and CBP- $12.28 \%$. After 6 months $88.68 \%$ of subjects with CWP and $72.38 \%$ with CBP participated in the follow-up screening. "Persistent" CWP was present in $79.43 \%$ and "persistent" CBP- in $47.37 \%$ of respondents. The majority of subjects with CWP (98.08\%) and CBP $(88.89 \%)$ consulted the general practitioner (GP) during the last year, mainly because of CMP. Many CWP (54.90\%) and CBP $(37.50 \%)$ sufferers consulted the GP 6-10 times during the last year. They were ordered a lot of investigations by the GP and were referred to specialists for medical advice (e.g., to a neurologist- $56.86 \%$ of CWP and $66.67 \%$ of CBP sufferers but to a rheumatologist- only $3.92 \%$ of CWP and $16.67 \%$ of CBP sufferers). The diagnosis was altered only in a very few cases. CBP was poorly diagnosed and fibromyalgia syndrome was not diagnosed at all by the GP. CMP patients were treated mainly with nonsteroidal anti-inflammatory drugs. No one received antidepressants, only $29.41 \%$ of CWP and $54.17 \%$ of CBP sufferers were prescribed rehabilitation procedures, but often they were hospitalised (27.45\% of CWP and $29.17 \%$ of CBP cases) during the last year. Quite a big number of CWP sufferers experienced either short-time or permanent disability.

Conclusion CMP syndromes are common in the outpatient population. More attention and priority for education of medical professionals concerning early diagnosis and appropriate treatment of CMP should be given by responsible health care authorities.

\section{SAT0233 A RANDOMISED COMPARISON OF CARE PROVIDED BY A CLINICAL NURSE SPECIALIST, INPATIENT TEAM CARE AND DAY PATIENT TEAM CARE IN RHEUMATOID ARTHRITIS (RA)}

${ }^{1} \mathrm{GJ}$ Tijhuis, ${ }^{2} \mathrm{AH}$ Zwinderman, ${ }^{1} \mathrm{JM}$ Hazes, ${ }^{3} \mathrm{WB}$ Van den Hout, ${ }^{1} \mathrm{FC}$ Breedveld, ${ }^{1} \mathrm{TP}$ Vliet Vlieland. ${ }^{1}$ Rheumatology; ${ }^{2}$ Medical Statistics; ${ }^{3}$ Medial Decision Making, Leiden University Medical Centre, Leiden, Netherlands

10.1136/annrheumdis-2001.869

Background The role of clinical nurse specialists in the management of patients with RA is evolving, and their effectiveness in comparison with care provided by a rheumatologist alone has been established. However, controlled studies showing how the effectiveness of clinical nurse specialists compares to that of multi-disciplinary team care, a treatment strategy that is considered to be optimal in chronic disease management, are lacking. Objectives The first aim of the study was to compare the clinical effectiveness of care delivered by a clinical nurse specialist with inpatient team care and day patient team care in patients with RA. The second aim was to determine factors predicting clinical response.

Methods A multi-centre, randomised controlled clinical trial including consecutive outpatients with RA and progressive functional limitations over the past 6 weeks. Clinical assessments recorded on study entry, weeks $6,12,26$ and 52 comprised the HAQ, MACTAR functional index, RAND-36 quality of life questionnaire, health utility rating scale (RS) and DAS. Patients? satisfaction with care was measured on a visual analogue scale. Within-group differences between baseline and follow-up scores were tested with the Wilcoxon signed rank matched-pairs test. Co-variance analysis was used to analyse between-group differences and to determine factors predicting clinical response.

Results 210 patients (median age 58 years, median disease duration 1.8 years and median HAQ score 1.25 ) were included in the study. Within all 3 groups functional status, quality of life, health utility and disease activity improved significantly over time (all P-values <0.05). A comparison of clinical outcome among the three groups and a comparison between the nurse specialist group and the inpatient and day patient care groups together did not show any sustained significant differences. Subgroup analysis showed that with increasing age, the most favourable outcome according to the HAQ shifted from care provided by a clinical nurse specialist and inpatient care to day patient care. Patients? satisfaction with care was significantly lower in the nurse specialist group than in the inpatient and day patient care groups $(\mathrm{P}<0.001)$.

Conclusion Care provided by a clinical nurse specialist appears to have a similar clinical outcome in comparison with inpatient and day patient team care in patients with RA. Age was the only factor related to differences in functional outcome between the three treatment groups. The choice of management strategy may, 